\title{
SATURN KILOMETRIC RADIATION NEAR A SOURCE CENTER ON DAY 73, 2008
}

\author{
J. D. Menietti*, R. L. Mutel*, P. Schippers*, S.-Y. Ye*, O. Santolik ${ }^{\dagger}$, \\ W.S. Kurth*, D. A. Gurnett*, L. Lamy ${ }^{\ddagger}$, and B. Cecconi ${ }^{\ddagger}$
}

\begin{abstract}
The Cassini spacecraft flew very near a source region of Saturn kilometric radiation (SKR) on day 073 of 2008 . This is the second known encounter with a source region at high latitude. The radio and plasma wave instrument, RPWS, observed intense SKR in the extraordinary (X) mode. The electron low-energy spectrometer (ELS) obtained a phase space distribution of sufficient energy and pitch angle resolution to allow growth rate calculations for the observed wave emissions. There is evidence of a shell or horseshoe electron plasma distribution that is unstable to the growth of SKR via the cyclotron maser instability. We present results of these calculations for comparison with a previously reported source region encounter.
\end{abstract}

\section{Introduction}

Auroral radio emissions are observed at all the magnetized planets (Earth, Jupiter, Saturn, Uranus, and Neptune, cf Zarka et al. [1998]). The primary source of these radio emissions is believed to be the cyclotron maser instability (CMI)[Wu and Lee, 1979]. The free energy source has more recently been identified as trapped electrons [Louarn and LeQueau, 1996] and electron shell or horseshoe distributions with energies in the range 1 to $10 \mathrm{keV}$ [Delory et al., 1998; Su et al., 2007]. The CMI as applied to terrestrial auroral kilometric radiation (AKR) has been articulated by Wu and Lee [1979] to explain emission occurring near the local cyclotron frequency, $\mathrm{f}_{c e}$. The ratio of plasma frequency, $\mathrm{f}_{p}$, to $\mathrm{f}_{c e}$ must be small. The emission propagates at large wave normal angle primarily in the right-hand extraordinary (RX) mode. All of these theoretical predictions have been confirmed by observations of the FAST satellite [cf. Ergun et al., 1998], and by numerical simulations [cf. Pritchett et al., 2002].

\footnotetext{
* Department of Physics and Astronomy, University of Iowa, Iowa City, IA 52242, USA

† Department of Space Physics, Institute of Atmospheric Physics, Prague, Czech Republic, and Faculty of Mathematics and Physics, Charles University, Prague, Czech Republic

‡LESIA, Observatoire de Paris, UMR CNRS 8109, 92195 Meudon, France
} 
While auroral emission has been observed from the magnetized planets and the CMI is generally accepted as the primary source of this emission, until recently only at Earth has the source region been directly observed by spacecraft. Kurth et al. [2011], Lamy et al. [2010], and Schippers et al. [2011] have recently reported observations within or very near an auroral source region at Saturn. This encounter occurred on 17 October 2008 during a period when Cassini was at high orbital inclination, and surprisingly, was at a local time near midnight.

The Cassini spacecraft flew a series of orbits at high inclination and latitudes during 2008. These orbits had periapses over the southern poleward regions of Saturn from a few to 5 Saturn radii $\left(\mathrm{R}_{s}=60,268 \mathrm{~km}\right)$. SKR is observed typically in the frequency range from $\sim 30$ $\mathrm{kHz}$ to $\sim 1 \mathrm{MHz}$, but rarely at frequencies as low as $10 \mathrm{kHz}$, which would require source regions extending from near the surface to as high as $5 \mathrm{R}_{s}$. Encounters with SKR source regions were not to be expected except under unusual conditions such as periods of solar wind compression that are associated with SKR intensifications and extensions to lower frequency as explained in Kurth et al. [2005], Jackman et al. [2009; 2010], Bunce et al. [2010]. This was probably the case for the SKR source encounter reported by Kurth et al. [2011], Lamy et al. [2010], and Mutel et al. [2010]. Mutel et al. [2010] have reported a calculation of the growth rate of the SKR using electron phase space distributions measured in situ by the electron spectrometer (ELS), reporting a weak shell distribution. These calculations confirmed again the CMI as the likely source mechanism and agree with the terrestrial observations of Ergun et al. [1998]. After an extensive search of the SKR data obtained by the Cassini RPWS, several other examples of SKR source region candidates have been found, but only one with sufficient electron phase space density to warrant a serious investigation of wave growth. In this paper we extend the study of Mutel et al., 2010] by investigating the electron distribution and radio emission spectra for a similar, but not as ideal event. By comparing the data for the similar events we attempt to draw some general conclusions about the plasma environment and the free energy source of SKR.

\section{Instrumentation, Observations, and Analysis}

The Cassini Radio and Plasma Wave Science (RPWS) instrument measures oscillating electric fields over the frequency range $1 \mathrm{~Hz}$ to $16 \mathrm{MHz}$ and magnetic fields in the range 1 $\mathrm{Hz}$ to $12 \mathrm{kHz}$ [cf. Gurnett et al., 2004]. The instrument uses 3 nearly orthogonal electric field antennas and 3 orthogonal magnetic search coil antennas, providing a directionfinding capability. There are 5 receiver systems, including a high frequency receiver (HFR) covering $3.5 \mathrm{kHz}$ to $16 \mathrm{MHz}$; a medium frequency receiver (MFR) covering $24 \mathrm{~Hz}$ to $12 \mathrm{kHz}$; a low frequency receiver (LFR) covering $1 \mathrm{~Hz}$ to $26 \mathrm{~Hz}$; a 5-channel waveform receiver operating in either a $1-26 \mathrm{~Hz}$ or $3 \mathrm{~Hz}-2.5 \mathrm{kHz}$ mode. The data presented in this study are measured by the LFR, MFR, and HFR.

The Cassini Plasma Spectrometer (CAPS) is composed of three sensors: the electron spectrometer (ELS), the ion beam spectrometer (IBS), and the ion mass spectrometer (IMS). Of importance in this study is the ELS that contains an 8-detector fan array in a single plane. Each detector has a $5.2^{\circ} \times 20^{\circ}$ field of view for a total in-plane field of view 
of $160^{\circ}$. The instrument measures electron energy from $0.6 \mathrm{eV}$ to $28,250 \mathrm{eV}$ over a period of 2 seconds with a resolution of $\delta \mathrm{E} / \mathrm{E}=0.17$ [cf. Young et al., 2004].

In Figure 1a (top) we present a frequency-time spectrogram of the RPWS plasma wave data for day 73 of 2008 between 04:15 - 05:05 UT. The frequency scale is linear and the gray bar indicates the electric field intensity. Intense SKR is seen throughout this interval extending from approximately $4 \mathrm{kHz}$ to over $10 \mathrm{kHz}$, and importantly, extending below the local measured electron cyclotron frequency (shown by the white line), particularly in the interval from about 04:35 to 04:40 (indicated by hash marks on the horizontal axis). The SKR spectral densities are similar in intensity to those observed on October 17, 2008. It is this time period in which the satellite could be within the SKR source region since SKR is a gyroresonant emission, but the RPWS frequency resolution is not sufficient to definitely confirm this. Figure $1 \mathrm{~b}$ (bottom) presents the circular polarization results for the same time period shown in Figure 1a (note the time axes are not perfectly aligned). The dominate polarization sense of SKR from sources in the southern hemisphere is LH; The LH polarization is represented by the lighter shades (1.0) while RH polarization is represented by the darker shades (-1.0). Between 4.6 to 5.0 hrs UT note that SKR emission is in lighter shades and extends to $\mathrm{f}<\mathrm{f}_{c e}$. See Ye et al. [2010] and Fischer et al. [2009] for a description of how the polarization measurements are obtained. We have examined the electron phase space distribution (PSD) function from the ELS instrument for the time period from about 04:35 to 04:40 UT. During this time the instrument required several minutes to obtain a full range of pitch angle samples. However, this period of time is much longer than the growth period of SKR, and the electron distribution function observed after a minute of time is relaxed due to growth of the waves. To overcome this problem we chose a 16-second sampling period which provided a balance between sufficient time resolution of the PSD and a sampling of the pitch angles. In Figure 2 we display a plot of a sampling of the PSD versus total velocity $\left(\sqrt{v_{\|}^{2}+v_{\perp}^{2}}\right)$ for a 16 -second time interval commencing at 04:35:48. Each line represents data for a different anode (pitch angle), ranging from 50.6 to $99.25^{\circ}$. Note that there are a number of anodes (see figure caption for pitch angles) with intensity enhancements that occur near 4-6 $\times 10^{7} \mathrm{~m} / \mathrm{s}$, indicating a shell-like distribution. All anodes show some enhancement for $\mathrm{V}_{t o t}>4 \times 10^{7}$ except anode 1 for a pitch angle of 86.5 degrees (lowest solid curve). To model this distribution we introduce a Dory-Guest-Harris (DGH) model distribution [Dory et al., 1965], analytical function as

$$
f(p)=\frac{n}{j !(2 \pi)^{3 / 2} V_{\perp}^{2} V_{\|}}\left(\frac{v_{\perp}^{2}}{2 V_{\perp}^{2}}\right)^{j} \exp \left[-\frac{v_{\perp}^{2}}{2 V_{\perp}^{2}}-\frac{\left(v_{\perp}-U\right)^{2}}{2 V_{\perp}^{2}}\right]
$$

where $\mathrm{n}$ is the number density, $\mathrm{V}$ is the thermal velocity, $U$ is the drift velocity, and $\mathrm{j} \geq 0$ is an integer. Parallel and perpendicular are relative to the magnetic field. For $\mathrm{j}=0$ the distribution is a bi-Maxwellian. In Figure 3 we plot the data for anode 5 (pitch angle $=50.6^{\circ}$, asterisks) and the model DGH distribution for the warm component only (crosses). The warm component has $\mathrm{n}_{w}=1.9 \times 10^{-4} \mathrm{~cm}^{-3}, \mathrm{~V}_{\|}=3.5 \times 10^{7} \mathrm{~m} / \mathrm{s}, \mathrm{V}_{\perp}=1.1 \times$ $10^{7} \mathrm{~m} / \mathrm{s}$, and $\mathrm{j}=10$, which places the center of a broad model shell distribution near 7.4 $\mathrm{keV}$. Reasonable fits of the data also occur for the approximate range $1.3<\mathrm{n}_{w}<2.0 \times$ $10^{-4} \mathrm{~cm}^{-3}$. The total plasma density, $\mathrm{n}_{e}$, is estimated by the upper cutoff frequency of the 


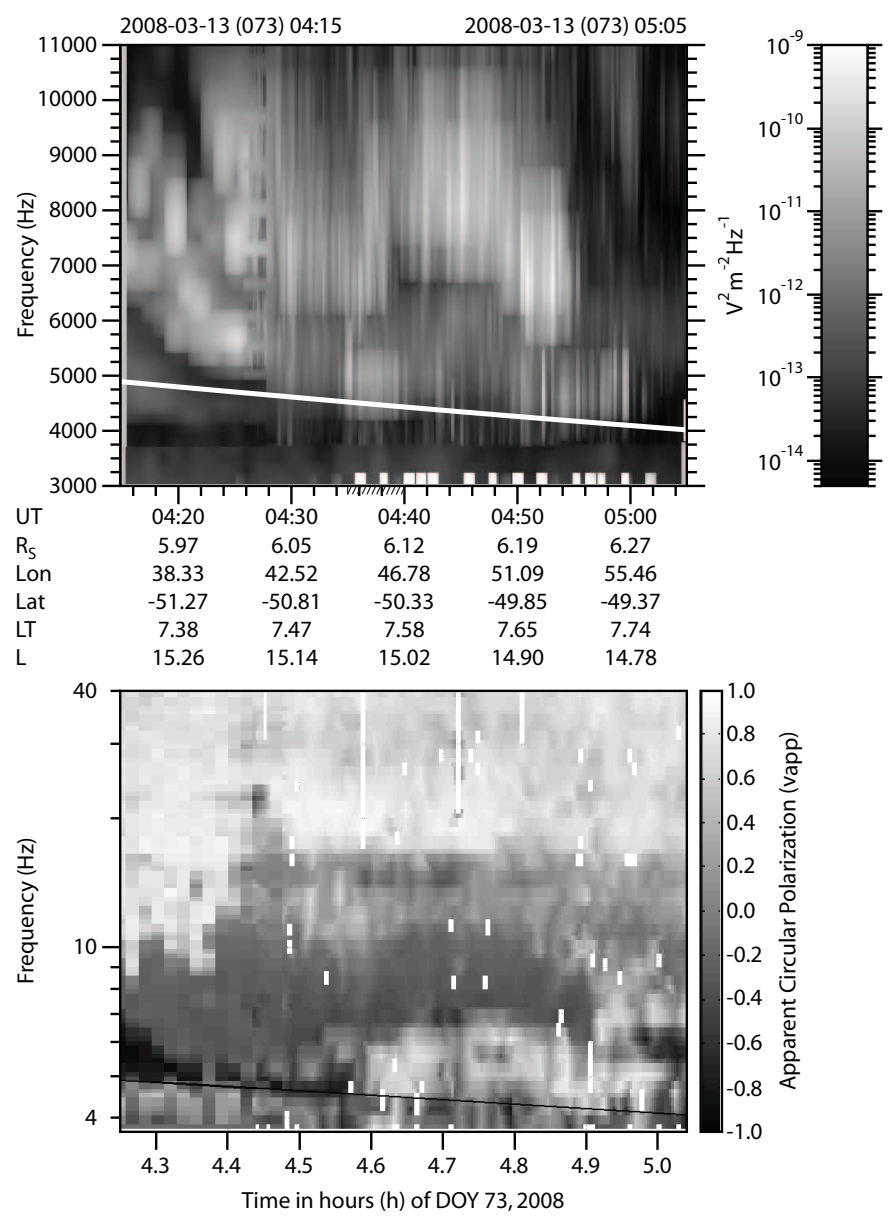

Figure 1: (1a, top) Frequency-time spectrogram of RPWS plasma wave data for day 73 of 2008 between 04:15-05:05 UT. The frequency scale is linear and the gray bar indicates electric field intensity. (1b, bottom) Apparent circular polarization results for a time period including that shown in Figure 1a (note the time axes are not perfectly aligned). LH polarization is represented by lighter shades (1.0) while RH polarization is represented by darker shades (-1.0). Between 4.6 to 5.0 hrs UT note that SKR emission is in lighter shades and extends to $f<f_{c e}$.

whistler mode emission which is close to $300 \mathrm{~Hz}$. For $\mathrm{n}_{w}=1.9 \times 10^{-4} \mathrm{~cm}^{-3}, \mathrm{n}_{w} / \mathrm{n}_{e}=0.4$. The cold core distribution is included in the fit, but the observed low-energy plasma 


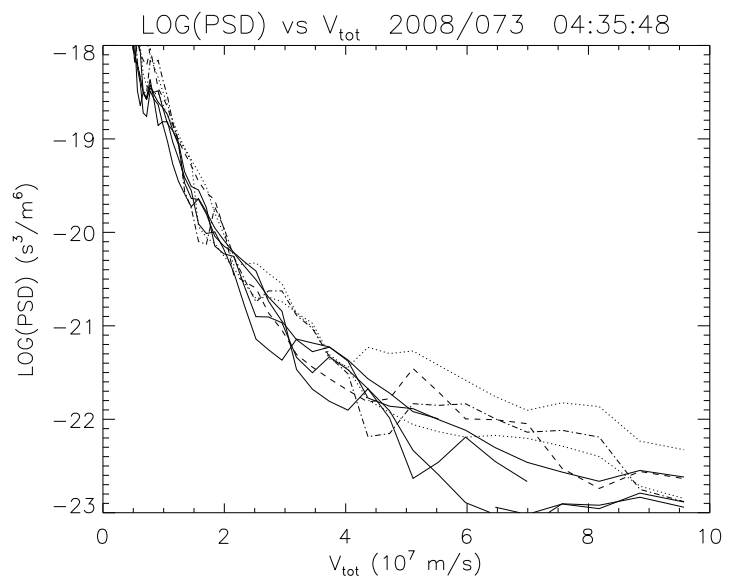

Figure 2: A plot of a sampling of the PSD versus total velocity $\left(\sqrt{\left(v_{\|}^{2}+v_{\perp}^{2}\right.}\right)$ for a 16 -second time interval commencing at 04:35:48. Each line represents data for a different anode (pitch angle), ranging from 50.6 to $99.25^{\circ}$. Anode 5 (50.6 / dashed); anode 7 (58.5 $/$ upper dotted); anode 4 (54.9 $/$ dot-dash); anode 0 (99.25\%/lower dotted).

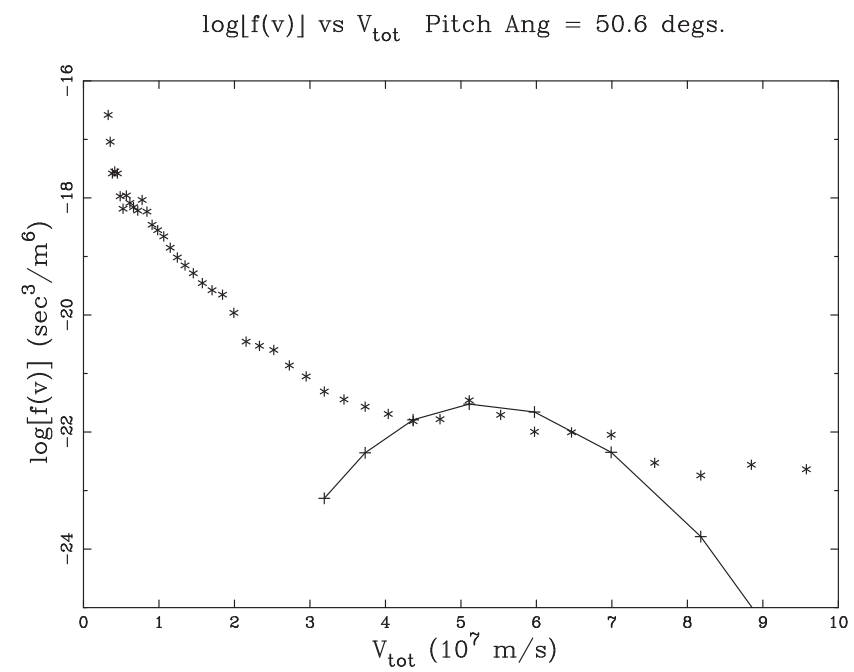

Figure 3: A plot of the data for anode 5 (pitch angle $=50.6^{\circ}$, asterisks) and the model DGH distribution for the warm component only (crosses).

contains photoelectrons and trapped electrons due to spacecraft charging. The fit of the model for the shell distribution near $\mathrm{V}_{t o t}=4-5 \times 10^{7} \mathrm{~m} / \mathrm{s}$ is important to the growth rate calculations in the CMI theory, because it lies near the resonance circle. 
The growth rate for the $\mathrm{X}$-mode in the $\mathrm{CMI}$ is given in a simple form as

$$
\gamma=\frac{\omega_{i}}{\Omega}=\frac{\pi^{2}}{4} \frac{n_{\omega}}{n_{e}^{2}} \beta^{2} c^{2} v_{r}^{2} \int_{0}^{\pi} d \psi \sin ^{2} \psi\left[\frac{\partial f}{\partial v_{\perp}}+\frac{k_{\|} v_{\perp}}{\Omega_{c e}} \frac{\partial f}{\partial v_{\|}}\right]_{|\mathbf{v}|=v_{r}}
$$

where $\omega_{i}$ is the imaginary part of the angular frequency, $\mathrm{n}_{w}$ is the density of warm plasma, $\Omega_{c e}$ is the electron angular cyclotron frequency, $\beta$ is the ratio of electron plasma frequency to electron cyclotron frequency, and $f$ is the electron distribution function, and $\mathrm{k}_{\|}$is the parallel component of the wave normal vector. The integration path is along the resonance ellipse, which for $\mathrm{v} \ll \mathrm{c}$ is a circle given by

$$
v_{r}=\left(v_{c}^{2}-2 c^{2} \delta \omega\right)^{1 / 2}
$$

with $\mathrm{v}_{c}=\mathrm{k}_{\|} \mathrm{c}^{2} / \Omega_{c e}$ and $\delta \omega=\left(\omega-\Omega_{c e}\right) / \Omega_{c e}$. At this time $\mathrm{f}_{c e}=4500 \mathrm{~Hz}$. Results of growth rate calculations for the RX mode are performed for a range of wave normal angles and ratios $\mathrm{f} / \mathrm{f}_{c e}$ and are displayed in a 2-dimensional contour plot in Figure 4. Strongest growth occurs for wave normal angles extending over $20^{\circ}$ and for ratios $0.990<\mathrm{f} / \mathrm{f}_{c e}<0.993$. The stepped nature of the plot results from finite iterations of the parameters. The growth rates are comparable to those determined by Mutel et al. [2010].

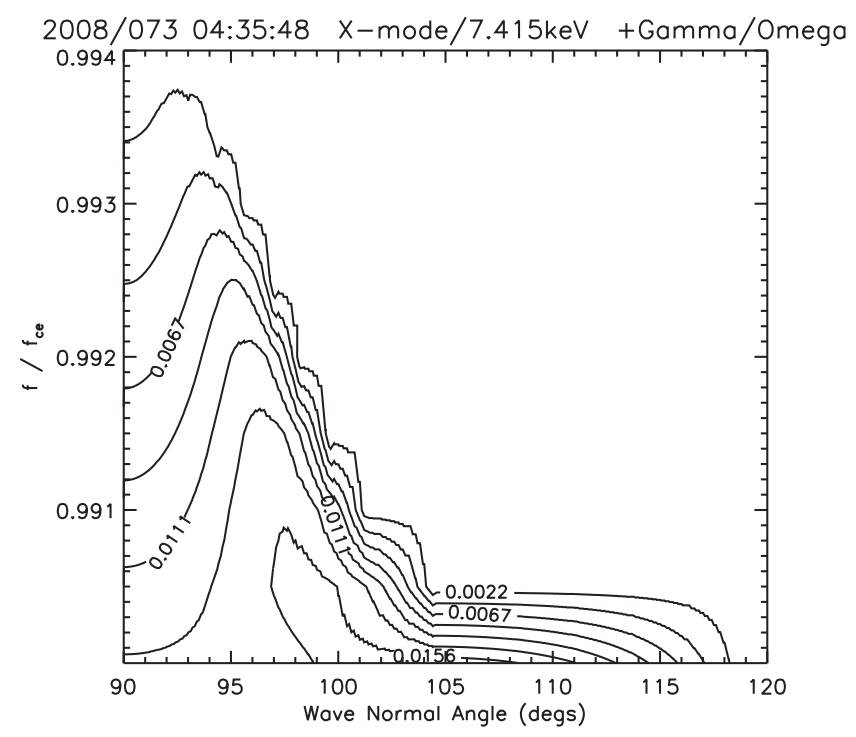

Figure 4: Contours of positive growth rate $\left(\gamma / \Omega_{c e}\right)$ for the $R X$ mode are plotted as a function of normalized frequency and wave normal angle. Growth is strongest for wave normal angles $<100^{\circ}$.

To estimate the power gain of the observations relative to the sky background and to the calculated gain we refer to the discussion in section 2.3 of Mutel et al. [2010]. From 
Figure 1a we note that the intensity of the observed electric fields near the source region is $\sim 10^{-11} \mathrm{~V}^{2} /\left(\mathrm{m}^{2} \mathrm{~Hz}\right)$ or equivalently about $1.7 \times 10^{-15} \mathrm{~W} /\left(\mathrm{m}^{2} \mathrm{~Hz}\right)$, and we estimate the background intensity is $\sim 6 \times 10^{-24} \mathrm{~W} /\left(\mathrm{m}^{-2} \mathrm{~Hz}\right)$, yielding an observed gain of $\mathrm{G}_{\text {obs }} \sim$ $3 \times 10^{8}$. In estimating the gain from the CMI we write $\mathrm{G}_{C M I}=\exp \left[\left(2 \omega_{i} \mathrm{~L}_{c}\right) / \mathrm{V}_{g}\right]$, where $\mathrm{L}_{c}$ is the convective growth length assumed to be $\sim 1000 \mathrm{~km}$, and $\mathrm{V}_{g}$ is the group velocity, which we assume to be $\sim 0.1 \mathrm{c}$ (cf. Mutel et al., 2010). For $\omega_{i} \sim .01-.02 \omega_{c e}$ these substitutions yield $\mathrm{G}_{C M I} \sim 1.5-3.0 \times 10^{8}$ which is comparable to the observed gain.

\section{Summary and Conclusions}

Discovery of a second possible SKR source region has allowed the comparison of observations and an estimate of SKR growth rates. The wave observations present a brief encounter (several minutes) with an SKR source region near a local time of 8 hours at a latitude of $\sim-50^{\circ}$. This is close to the nominal local time of SKR source regions as reported by Lamy et al. [2009]. The intensity levels for this event are not as high as for the previously reported event on day 291 of 2008 [Lamy et al., 2010]. The pitch angle coverage of the ELS instrument is not as good as for the event of day 291, but the observations suggest a shell-like electron distribution centered near $7 \mathrm{keV}$. Based on assumptions outlined above, the modeled electron distribution is adequate to generate $\mathrm{X}$ mode emission at the intensity level of the observations. We are hopeful that more source encounters will be experienced before the end of the mission to continue these investigations.

Acknowledgments. Research at the U. of Iowa was supported by NASA through contract 1415150 with the Jet Propulsion Laboratory.

\section{References}

Bunce, E., S.W.H. Cowley, D. L. Talboys, M.K. Dougherty, L. Lamy, W.S. Kurth, P. Schippers, B. Cecconi, P. Zarka, C. S. Arridge, and A. J. Coates, Extraordinary field-aligned current signatures in Saturn's high-latitude magnetosphere: Analysis of Cassini data during Revolution 89, J. Geophys. Res., 115, A10238, doi:10.1029/2010JA015612, 2010.

Cecconi, B., P. Zarka, and W.S. Kurth, SKR polarization and source localization with the Cassini/RPWS/HFR instrument: First results, in Planetary Radio Emissions VI, edited by H. O. Rucker, W. S. Kurth, and G. Mann, 37-49, Austrian Academy of Sciences Press, Vienna, 2006.

Delory, G. T., R. E. Ergun, C. W. Carlson, L. Muschietti, C. C. Chaston, W. Peria, and J.P. McFadden, FAST observations of electron distributions within AKR source regions, Geophys. Res. Lett., 25, 12, 2069-2072, 1998.

Dory, R. A., G. E. Guest, and E. G. Harris, Unstable electrostatic plasma waves propagating perpendicular to a magnetic field, Phys. Rev. Lett., 14, 131-133, doi:10.1103/ PhysRevLett.14.131, 1965. 
Ergun, R. E., et al. (18 co-authors), FAST satellite wave observations in the AKR source region, Geophys. Res. Lett., 25, 12, 2061-2064, 1998.

Fischer, G., B. Cecconi, L. Lamy, S.-Y. Ye, U. Taubenschuss, W. Macher, P. Zarka, W.S. Kurth, and D. A. Gurnett, Elliptical polarization of Saturn Kilometric Radiation Observed from High Latitudes, J. Geophys. Res., 114, A08216, doi:10.1029/2009JA014176, 2009.

Gurnett, D. A., et al. (29 co-authors), The Cassini radio and plasma wave investigation, Space Sci. Rev., 114, 395-463, 2004.

Jackman, C. M., L. Lamy, M. P. Freeman, P. Zarka, B. Cecconi, W. S. Kurth, S. W. H. Cowley, and M. K. Dougherty, On the character and distribution of lower-frequency radio emissions at Saturn and their relationship to substorm-like events, J. Geophys. Res., 114, A08211, doi:10.1029/2008JA013997, 2009.

Jackman, C. M., C. S. Arridge, J. A. Slavin, S. E. Milan, L. Lamy, M. K. Dougherty, and A. J. Coates, In situ observations of the effect of a solar wind compression on Saturn's magnetotail, J. Geophys. Res., 115, A10240, doi:10.1029/2010JA015312, 2010.

Kurth, W. S., D. A. Gurnett, J. T. Clarke, P. Zarka, M. D. Desch, M. L. Kaiser, B. Cecconi, A. Lecacheux, W. M. Farrell, P. Galopeau, J.-C. Gérard, D. Grodent, R. Prangé, M. K. Dougherty, and F. J. Crary, An Earth-like correspondence between Saturn's auroral features and radio emission, Nature, 433, 722-725, doi:10.1038/nature03334, 2005 .

Kurth, W.S., et al. (20 co-authors), A close encounter with a Saturn kilometric radiation source region, in Planetary Radio Emissions VII, edited by H.O. Rucker, W.S. Kurth, and G. Fischer, this issue, 75-85, Austrian Academy of Sciences Press, Vienna, 2011.

Lamy, L., P. Zarka, B. Cecconi, R. Prange, W. S. Kurth, and D. A. Gurnett, Saturn kilometric radiation: Average and statistical properties, J. Geophys. Res., 113, A07201, doi:10.1029/2007JA012900, 2008.

Lamy, L., B. Cecconi, R. Prange, P. Zarka, J. D. Nichols, and J. T. Clarke, An auroral oval at the footprint of Saturn's kilometric radio sources, colocated with the UV aurorae, J. Geophys. Res., 114, A10212, doi:10.1029/2009JA014401, 2009.

Lamy, L., P. Schippers, P. Zarka, B. Cecconi, C. S. Arridge, M. K. Dougherty, P. Louarn, N. André, W.S. Kurth, R. L. Mutel, D. A. Gurnett, and A. J. Coates, Properties of Saturn kilometric radiation measured within its source region, Geophys. Res. Lett., 37, L12104, doi:10.1029/2010GL043415, 2010.

Louarn, P., and D. Le Queau, Generation of the auroral kilometric radiation in plasma cavities-II. The cyclotron maser instability in small size sources, Planet. Space Sci., 44, No. 3, 211-224, 1996.

Mutel, R. L., J. D. Menietti, D. A. Gurnett, W. Kurth, P. Schippers, C. Lynch, L. Lamy, C. Arridge, and B. Cecconi, CMI growth rates for Saturnian kilometric radiation, Geophys. Res. Lett., 37, L19105, doi:10.1029/ 2010GL044940, 2010. 
Pritchett, P. L., R. J. Strangeway, R. E. Ergun, and C. W. Carlson, Generation and propagation of cyclotron maser emissions in the finite auroral kilometric radiation source cavity, J. Geophys. Res., 107, A12, 1437, doi:10.1029/2002JA009403, 2002.

Schippers, P., C. S. Arridge, J. D. Menietti, D. A. Gurnett, L. Lamy, B. Cecconi, D. G. Mitchell, N. Andre, W.S. Kurth, S. Grimald, M. K. Dougherty, A. J. Coates, and D. T. Young, Auroral electron distributions within and close to the Saturn kilometric radiation source region, J. Geophys. Res., 166, A5, A05203, doi:10.1029/ 2011JA016461, 2011.

Su, Y.-J., R. E. Ergun, S. T. Jones, R. J. Strangeway, C. C. Chaston, S. E. Parker, and J.L. Horwitz, Generation of short-burst radiation through Alfvenic acceleration of auroral electrons, J. Geophys. Res., 112, A06209, doi:10.1029/2006JA012131, 2007.

Wu, C.S., and L. C. Lee, A theory of terrestrial kilometric radiation, Astrophys. J., 230, 621-626, doi:10.1086/157120, 1979.

Ye, S.-Y., J. D. Menietti, G. Fischer, Z. Wang, B. Cecconi, D. A. Gurnett, and W. S. Kurth, $\mathrm{Z}$ mode waves at the source of Saturn narrowband radio emissions, J. Geophys. Res., 115, A08228, doi:10.1029/2009JA015167, 2010.

Young, D. T., et al. (57 co-authors), Cassini plasma spectrometer investigation, Space Sci. Rev., 114, 1-112, 2004.

Zarka, P., Auroral radio emissions at the outer planets: Observations and theories, J. Geophys. Res., 103, 20, 159-20,194, doi:10.1029/98JE01323, 1998. 
